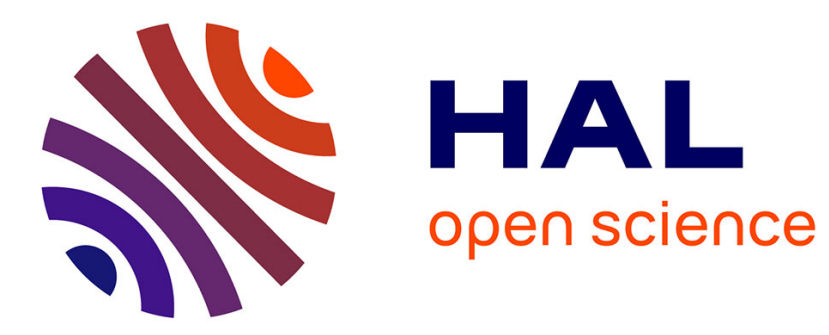

\title{
Exploiting Natural Dynamics in order to Increase the Feasible Static-Wrench Workspace of Robots
}

Rafael Balderas Hill, Sébastien Briot, Abdelhamid Chriette, Philippe Martinet

\section{To cite this version:}

Rafael Balderas Hill, Sébastien Briot, Abdelhamid Chriette, Philippe Martinet. Exploiting Natural Dynamics in order to Increase the Feasible Static-Wrench Workspace of Robots. The 15th IFToMM World Congress, Jun 2019, Cracovie, Poland. hal-02003103

\section{HAL Id: hal-02003103 https://hal.science/hal-02003103}

Submitted on 24 Jun 2019

HAL is a multi-disciplinary open access archive for the deposit and dissemination of scientific research documents, whether they are published or not. The documents may come from teaching and research institutions in France or abroad, or from public or private research centers.
L'archive ouverte pluridisciplinaire HAL, est destinée au dépôt et à la diffusion de documents scientifiques de niveau recherche, publiés ou non, émanant des établissements d'enseignement et de recherche français ou étrangers, des laboratoires publics ou privés. 


\title{
Exploiting Natural Dynamics in order to Increase the Feasible Static-Wrench Workspace of Robots
}

\author{
Rafael Balderas Hill ${ }^{1,2}$, Sébastien Briot ${ }^{2,3}$, Abdelhamid Chriette ${ }^{1,2}$, and \\ Philippe Martinet ${ }^{4}$ \\ 1 École Centrale de Nantes, 44321 Nantes, France \\ 2 Laboratoire des Sciences du Numérique de Nantes (LS2N), UMR CNRS 6004, \\ 44321 Nantes, France \\ 3 Centre National de la Recherche Scientifique (CNRS) \\ 4 Centre de Recherche Inria Sophia Antipolis, 06902 Sophia Antipolis, France
}

\begin{abstract}
The reachable workspace of a robot carrying a payload is usually limited by the maximal value of the torque that each actuator can deliver. This results in limiting the zones for the robot to operate with the payload due to a possible division of its static-wrench workspace into several disconnected aspects.

In order to increase the reachable workspace areas, this paper proposes to exploit the natural oscillations in dynamics, so that the robot can carry a payload which is out of its feasible static-wrench workspace, i.e. to perform motions between two disconnected aspects, while constraining the torques of the actuators. This is done thanks to the solution of a boundary value problem, which seeks to smartly exchange the gravity potential energy and the kinetic energy in order to connect two desired payload positions, which are placed in two disconnected aspects. Simulations of the suggested approach on a 2-degree-of-freedom robot are performed and show the efficiency of the proposed approach.
\end{abstract}

Keywords: static-wrench workspace, natural dynamics, shooting method

\section{Introduction}

It is well-known that in several industrial robotic processes, robot manipulators are required to have as large as possible workspace. Nevertheless, when for some specific tasks, the robots are required to carry heavy payload objects, the workspace can be limited by the actuator maximum torques. The remaining workspace, limited by the torque limits, is called feasible static-wrench workspace, and it is generally defined by the set of all feasible end-effector poses for which the platform with payload can be reached statically, i.e. taking into

This work was conducted with the support of the Mexican Council for Science and Technology (CONACYT). 
account only the gravity effects [1][2][3]. As a consequence, if the maximal values of the actuator torques are not sufficiently high for a given payload, the reachable robot workspace will be reduced. This will restrict the zones where the robot can place the payload, and it may lead to disconnect the robot workspace into several aspects.

Most of the techniques developed for increasing static-wrench workspace feasibility have been applied for cable-driven parallel robots (CDPRs), in which the cables have been used as passive elements to find dynamic trajectories that seek to match the free response of the system with the desired payload motions [4][5][6][7]. The main idea is to replace the CDPR motor-cable arrangements with linear springs, from which an undamped mass-spring system is approximated. Then, the natural frequencies from such passive mechanical system are computed, and periodic trajectories are derived. Moreover, the natural frequencies of the virtual spring-based passive system are used for exploiting the dynamics of the system in order to find natural free-motion trajectories, thus increasing the feasible static-wrench workspace. Even if such techniques have shown their effectiveness when working with CDPRs, it is not the same case for rigid-link robots, in which no elastic element can be considered unless a spring is added in parallel to the active joints, as it is done in [8][9][10]. In those works, torsional springs are placed in parallel to the actuators, and they are used as energy storage in order to exploit the natural oscillations of pick-and-place robots in order to put the system near resonance, thus considerably decreasing the input torques.

Contrary to what it is done in [8][9][10], in which the spring potential energy is used to exploit the natural dynamics of the system, in this paper we propose to keep the robot unmodified, i.e. without any additional elastic element, and instead, to exploit the natural dynamics by using the payload potential energy. This will allow to increase the robot feasible static-wrench workspace thanks to a smart control of the natural oscillations in order to perform large amplitude motions between two disconnected aspects of the static-wrench workspace, similarly to the phenomenon occurring on an oscillating pendulum, in which all the energy is stored as potential energy when the payload is at the highest position of its swing, and it is then transferred continuously to kinetic energy.

This paper is divided as follows: Section 2 presents a recall on dynamic modeling of robot manipulators. Section 3 shows the algorithm formulation for performing optimal trajectories that connect two aspects of the static-wrench workspace, divided due to the maximal actuator torques. Finally, in order to show the effectiveness of the proposed approach, section 4 presents the results on a 2-DOF serial robot with different payloads and different maximal actuator torque values.

\section{Exploiting natural dynamics for enlarging feasible static-wrench workspace}

It is well-known that near natural frequencies of oscillatory systems, the actuator torques can be drastically reduced [8][9][10]. For instance, we know that, near 
natural frequencies of a spring-mass system, large oscillatory motions can be obtained while conserving low torques in the actuator, which leads to lowering the energy expenditure. This is possible because of a smart exchange of energies between the kinetic energy and the spring potential energy of the system.

Thus, in similar vein, in this section, we propose a strategy to smartly exploit the exchange between the gravity potential energy and the kinetic energy of a robot with payload, so that it can be put in near its free response, thus decreasing the input torques. In order to do so, we will firstly recall the dynamic modeling of robot manipulators. On a second stage, we will propose the formulation of a boundary value problem (BVP), which will allow us to compute the necessary optimal velocity, associated to the kinetic energy required to cross from one disconnected aspect to another with minimum effort. Then, similarly to an oscillating pendulum, thanks to the formulation of an optimisation problem, we will exploit the natural oscillations of the robot with payload to achieve the optimal velocity that match the system to its free response, obtained from the solution of the BVP.

\subsection{Physical Background}

Based on [11], let us briefly recall the dynamic modeling of robot manipulators. We will assume a kinematic architecture composed of $n$ degrees of freedom and driven by $n$ active joints. The position and velocity of the robot can be described by $\mathbf{q}$ and $\dot{\mathbf{q}}$ representing the $n$-dimensional vectors of active joint variables and of active joint velocities, respectively.

Then, by using the Lagrange formalism, the dynamic model of a robot manipulator can be written as follows:

$$
\boldsymbol{\tau}=\mathbf{M}(\mathbf{q}) \ddot{\mathbf{q}}+\mathbf{h}(\mathbf{q}, \dot{\mathbf{q}})+\mathbf{g}_{r}(\mathbf{q})+\mathbf{f}_{s}
$$

where $\boldsymbol{\tau}$ is an $n$-dimensional vector of the robot input efforts, $\mathbf{M}$ is an $(n \times n)$ definite positive matrix of inertia depending on the active joints coordinates $\mathbf{q}$. $\mathbf{h}$ is an $n$-dimensional vector of Coriolis and centrifugal effects and its value depends on the active joint coordinates $\mathbf{q}$, and their time derivatives $\dot{\mathbf{q}} \cdot \mathbf{g}_{r}$ is an $n$-dimensional vector grouping the gravitational effects. $\mathbf{f}_{s}$ is an $n$-dimensional vector grouping the active joint friction terms.

Based on the aforementioned computations, it is thus necessary to find a strategy to exploit the natural dynamics of (1), so that we can find free-motion trajectories that constrain the input torques grouped in $\boldsymbol{\tau}$ while performing large amplitude oscillations that connect aspects of the static-wrench workspace.

\subsection{Shooting method for solving Boundary Value Problems}

In the field of numerical analysis, a boundary value problem (BVP) is defined when, for solving a differential equation, two conditions are given at different values of the independent variable of the differential equation [12], i.e.:

$$
h^{\prime \prime}=f\left(t, h, h^{\prime}\right), \quad a<t<b
$$


with the boundary conditions defined by:

$$
h(a)=A, \quad h(b)=B
$$

where (2) is a second order differential equation, $t$ is the independent variable, $A$ and $B$ are the desired boundary conditions defined at $t=a$ and $t=b$ of the $\mathrm{BVP}$, respectively.

The classical way to solve the BVP (2)-(3), is by using the so-called shooting method [12], which treats the BVP as an initial value problem (IVP) [12], with the initial conditions given by:

$$
h(a)=A, \quad h^{\prime}(a)=\alpha
$$

where $\alpha$ represents the first derivative of $h(t)$ at $t=a$, or in other words, the slope of the solution, and the objective is to choose $\alpha$, so that the solution of (2) satisfies the remaining boundary condition $h(b)=B$ from (3). Practically speaking, the main idea of the shooting method is to numerically integrate (2), for different values of the slope $\alpha$, and then to take the solution of the differential equation that satisfies the boundary condition $h(b)=B$.

Thus, similarly, we propose to use a BVP formulation in order to find the optimal velocity $\dot{\mathbf{q}}_{A}$, or slope, associated to the necessary kinetic energy required to pass between two different disconnected aspects of the static-wrench workspace (See Fig. 1a).

\subsection{Shooting method applied on robot manipulators with heavy payloads}

To develop the BVP for the robot, we will denote $\dot{\mathbf{q}}_{A}$, as the optimal velocity at point $A$ in one disconnected aspect of the static-wrench workspace, that will allow to connect the aspects in point $B$ with null final velocity (See Fig. 1a).

In order to exploit the natural dynamics of the robot with payload to join two different aspects of the robot disconnected static wrench-workspace, in this section we propose to formulate a BVP, which can be solved with the shooting method [12]. The BVP formulation seeks to find the optimal velocity grouped in $\dot{\mathbf{q}}_{A}$, or slope, and associated to the optimal kinetic energy required to connect two aspects, that solve (1) for $\boldsymbol{\tau}=0$, while ensuring to meet the desired boundary positions defined in two disconnected aspects ( $A$ and $B$ in Fig. 1a), i.e.:

$$
\ddot{\mathbf{q}}=-\mathbf{M}^{-1}\left(\mathbf{h}(\mathbf{q}, \dot{\mathbf{q}})+\mathbf{g}_{r}(\mathbf{q})+\mathbf{f}_{s}\right)
$$

with the boundary conditions defined as the desired positions in two disconnected aspects of the feasible static-wrench workspace by $\mathbf{q}\left(t_{0}\right)=\mathbf{q}_{A}^{*}, \mathbf{q}\left(t_{f}\right)=\mathbf{q}_{B}^{*}$ with the formulation of the following position error vector:

$$
\mathbf{E}_{q}\left(\dot{\mathbf{q}}_{A}\right)=\mathbf{q}\left(t_{f}, \dot{\mathbf{q}}_{A}\right)-\mathbf{q}_{B}^{*}
$$

where $t_{f}$ is the final time, and $\dot{\mathbf{q}}$ and $\mathbf{q}$ are obtained from numerical single and double integration of $\ddot{\mathbf{q}}$, respectively. The shooting method is applied to 
iteratively find the proper joint velocities, grouped in $\dot{\mathbf{q}}_{A}$, such that expression (6) converge to zero as follows:

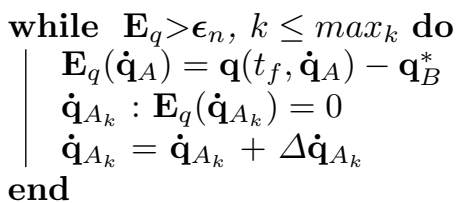

where $\dot{\mathbf{q}}_{A_{k}}=\dot{\mathbf{q}}_{A_{k+1}}$ representing the optimal set of joint velocities, $\max _{k}$ is the maximum number of iterations, and $\boldsymbol{\epsilon}_{n}$ is the error threshold.

Since we will consider that all motions of the robot will start from the equilibrium position of the system, i.e. lowest end-effector location, at $\mathbf{q}_{0}=[-\pi, 0]^{T}$, in the following section, we develop a strategy to optimally go from the equilibrium configuration velocity of the robot, to the optimal aspect-connecting velocity $\dot{\mathbf{q}}_{A}$.

\subsection{Initial oscillations for achieving optimal aspect-connecting velocity}

In the aforementioned formulation, we have presented a strategy based on a BVP to compute the optimal velocity $\dot{\mathbf{q}}_{A}$, associated to the kinetic energy required to join two disconnected aspects. Nevertheless, since we have chosen to start the motion from the equilibrium configuration of the robot $\left(\dot{\mathbf{q}}_{0}=\mathbf{0}\right)$, i.e. null initial stored potential energy $U_{0}$ to attain $\dot{\mathbf{q}}_{A}$, it is thus necessary to find a strategy to reach $\dot{\mathbf{q}}_{A}$ from rest state. One could think of planning a classical motion based on a fifth-degree polynomial which can fix the position, velocity and acceleration at the trajectory extremities, thus allowing to fix $\dot{\mathbf{q}}_{A}$. Nevertheless, since we seek to constrain the torques to operate within a range of motor limits, it is thus necessary to develop an optimal motion planner that integrates the effects of the dynamics to reach $\dot{\mathbf{q}}_{A}$. That is why, contrary to the aformentioned BVP formulation, which allows us to compute the kinetic energy required to connect boundary positions in different aspects, in this part we seek to propose an optimal strategy to attain that aspect-connecting velocity $\dot{\mathbf{q}}_{A}$. Similar to an oscillating pendulum, this can be done by exploiting the oscillations of the robot with payload in order to store the optimal payload potential energy to reach $\dot{\mathbf{q}}_{A}$. Thus, we propose to find such exciting initial oscillatory motions that accelerates the robot to reach $\dot{\mathbf{q}}_{A}$, while minimizing the input torques, by defining the following optimization formulation:

$$
\begin{array}{ll}
\text { minimize } & J=\int_{0}^{t_{f^{\prime}}} \sum_{i=1}^{n} \tau_{i}^{2} d t \\
\text { over } & \mathbf{x}_{\text {DecVar }} \\
\text { subject to } & \left|\tau_{i}\right| \leq \tau_{\max }, t_{\min } \leq t \leq t_{\max },\left|q_{i}\right| \leq q_{\max }
\end{array}
$$

where the decision variable vector $\mathbf{x}_{\mathbf{D}}$ ecVar contains the following parameters: $q_{i_{k}}, v_{i_{k}}, a_{i_{k}}$ and $t_{i_{k}}$. Similar to the strategy based on the via-point motion planner of [13], each of these parameters represents the amplitude, velocity, acceleration 


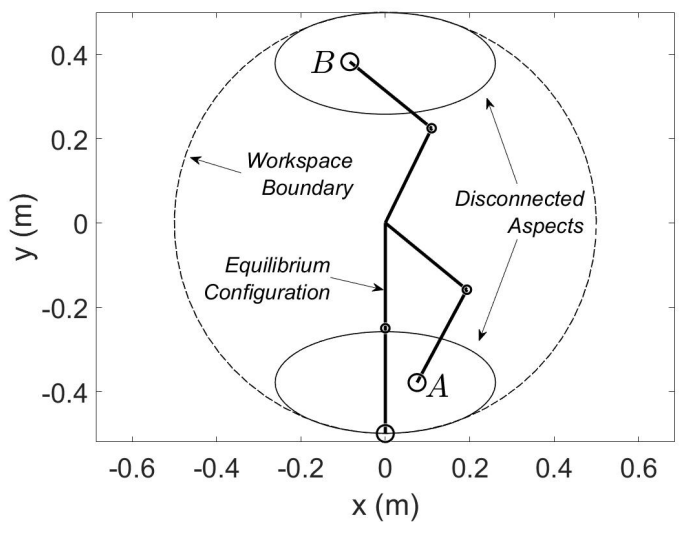

(a) Division of static-wrench workspace in different aspects

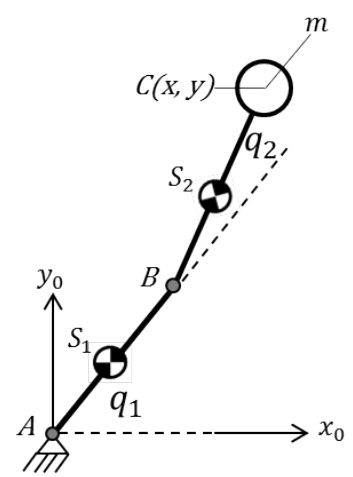

(b) Parameterization of $2 \mathrm{R}$ serial robot

Fig. 1: On the left, the graphical interpretation of the disconnection between aspects due to actuation limits on a $2 \mathrm{R}$ serial robot workspace. $A$ and $B$ represent the boundary conditions which seek to be joined thanks to the BVP. On the right, the kinematic structure of the 2-DOF robot with payload is shown

and time of oscillation for the exciting trajectories parameterized by using fifthdegree order polynomials, respectively (See Fig. 4, exciting oscillations). $k$ is an integer representing the number of required oscillations (via points) for attaining $\dot{\mathbf{q}}_{A}$. Finally, $t_{f}$ represents the final time of the optimization, and $\tau_{i}$ represents the torque of the actuated joints grouped in $\mathbf{q}$.

\section{Case Study}

\section{$3.1 \quad 2-D O F$ serial robot}

In this Section, numerical validations are made thanks to a co-simulation between Matlab and ADAMS in order to show the effectiveness of our approach. The proposed strategy for enlarging the feasible static-wrench workspace was validated on a 2 -DOF serial robot with payload, as shown in Fig. 1b. The active joint coordinates of the robot are parameterized by $\mathbf{q}=\left[q_{1}, q_{2}\right]^{T}$. The vector of payload pose is denoted as $\mathbf{x}=[x, y]^{T}$. The link lengths $l_{A B}$ and $l_{B C}$ are identical, and the center of mass of each link is located at a distance $l_{A S_{1}}$ and $l_{B S_{2}}$ from the rotation center of the motors $q_{1}$ and $q_{2}$, respectively. Moreover, the gravity field is directed along $\mathbf{y}_{0}$ and is equal to $\mathbf{g}=[0,-g, 0]^{T}, g>0$, being equal to $9.81 \mathrm{~m} / \mathrm{s}^{2}$. Each link mass is parameterized by $m_{1}$ and $m_{2}$, and the payload mass is denoted by $m$. Finally, the inertia of each link is given by $z z_{1}$ and $z z_{2}$, and it is measured about its center of mass $S_{1}$ and $S_{2}$, respectively.

The dynamic and geometric parameters for the simulationes, were defined by the following numerical values: inertia of the two links $z z_{1}=z z_{2}=0.133 \mathrm{~kg} / \mathrm{m}^{2}$, 
mass of each link $m_{1}=m_{2}=0.1 \mathrm{~kg}$, Coulomb friction terms $f_{s 1}=f_{s 2}=0.3$ $\mathrm{Nm}$, link lengths $l_{A B}=l_{B C}=0.25 \mathrm{~m}$ and the distance to center of mass for each link $l_{A S_{1}}=l_{B S_{2}}=0.125 \mathrm{~m}$. The dynamic equations for the 2 -DOF serial robot with payload are not given here due to page limitations. However, by using the Lagrange formalism shown in section 2.1, the expression for the robot can take the form (1).

\subsection{Results}

The algorithm proposed in section 2 was tested by defining two desired points in disconnected aspects of the static-wrench workspaces, as it is shown in the interpretation from Fig. 1a. The aim is to connect both separated aspects of the static-wrench workspace by solving the corresponding BVP. Moreover, in order to show the effectiveness of the proposed approach, four cases corresponding to different payloads, maximal actuator torques and desired boundary positions $(m$, $\tau_{\max }, A, B$, respectively) have been studied: Case 1: $m=0.5 \mathrm{~kg}, \tau_{\max }=1 \mathrm{Nm}$, $A=[0,-0.43], B=[0.05,0.4]$; Case $2: m=1 \mathrm{~kg}, \tau_{\max }=2 \mathrm{Nm}, A=[0.15,-0.4]$, $B=[0,0.4]$; Case $3: m=1.25 \mathrm{~kg}, \tau_{\max }=2.25 \mathrm{Nm}, A=[0,-0.45], B=[0,0.45]$; Case 4: $m=1.5 \mathrm{~kg}, \tau_{\max }=3 \mathrm{Nm}, A=[0,-0.45], B=[0.1,0.4]$.

Firstly, in Fig 2, the total static-wrench feasible workspace is computed for the case 2, i.e. all the configurations of the robot for which statically with the given payload, respects the actuator limits (only one case is shown due to page limitations). Moreover, a static torque map based on contours is shown in order to have an idea of the value, that in statics, the actuator torque must be able to deliver for each robot configuration. It is worth mentioning that a more accurate contour map could be computed based on [14], however, since for our case we have a simple system, we can easily compute the static torque at each position of the workspace. Once that the total static-wrench feasible workspace is computed, the algorithm proposed in section 2 is tested. The results in input torques for the four cases, for both BVP and exciting trajectories, are shown Table 1 . In addition to that, the optimal values $\dot{\mathbf{q}}_{A}$ from the BVP, for the different boundary conditions from the four cases, are shown as well in Table 1 . It can be seen that, based on the maximum value of the input torques, the efforts from the trajectory based on the shooting method are minimum, while the input efforts from the exciting trajectories are within the maximal motor torque values, thus constraining the actuator torques. In addition to that, Fig. 3 presents the input torques computed along the motion from case 2 in order to show the effectiveness of the approach to constrain the input efforts within a range of maximal actuator values that allows to join two disconnected aspects from the static-wrench workspace (only one case is shown due to page limitations). In Fig. 4 the optimal motions from the shooting method and from the exciting trajectories are shown in the workspace of the 2-DOF serial robot for case 2. It can be seen that thanks to the solution of the BVP, two points in different aspects of the divided staticwrench workspace can be joined while constraining the torques of the actuators. Furthermore, as it is shown in Table 1 and in Fig. 3, the input torques computed 


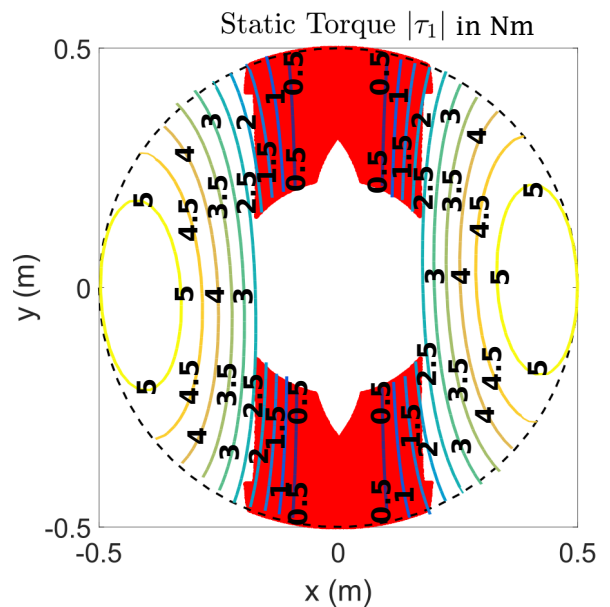

(a) Slice XY in which each contour represents a static torque for the first joint.

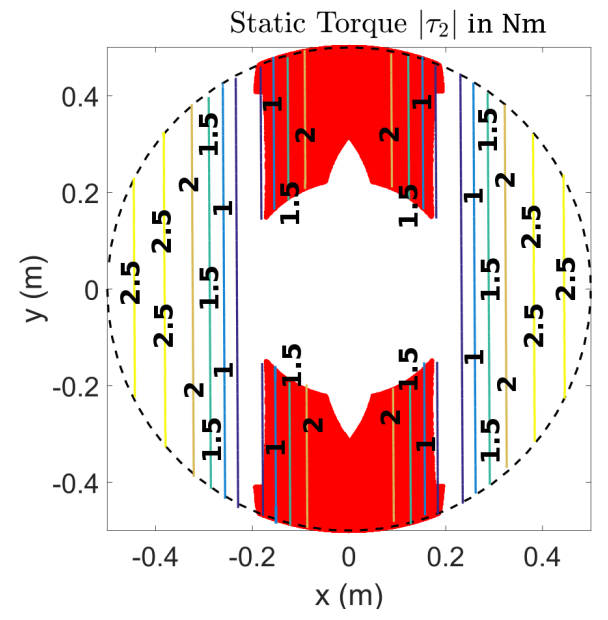

(b) Slice XY in which each contour represents a static torque for the second joint.

Fig. 2: In red, the total static-wrench feasible workspace for case 2 is shown, i.e. all configurations for which, the static torques are within the range of maximum torques $\left|\tau_{i}\right| \leq \tau_{\max }$. The center of both figures is empty due to the fact that a joint limit has been set for the second joint of $q_{2}=2.2 \mathrm{rad}$. Each contour in these figures represent a value for the static torque of the first and second joint, respectively, for the case 2 , i.e. a payload of $1 \mathrm{~kg}$ and a maximal actuator torque $\tau_{1_{\max }}=\tau_{2_{\max }}=2 \mathrm{Nm}$. Furthermore, the static torques contours are computed for all robot configurations within the workspace boundaries.

from the BVP are closed to zero, which means a correct optimal dynamics exploitation since we were seeking to solve (1) for $\boldsymbol{\tau}=0$. For a visualization of the results, a video of the co-simulations of the 2 -DOF serial robot connecting two different aspects, can be found in https://bit.1y/2QuLQdb

\section{Conclusions and future works}

This paper proposes a strategy for increasing the feasible static-wrench workspace for robots by exploiting the robot dynamics through the solution of a BVP which seeks to smartly control the free response of the system to perform motions that connect two different aspects of the static-wrench workspace. This has allowed to considerably increase the reachability to disconnected areas of the workspace of a 2-DOF serial robot with different payloads and for multiple desired boundary positions. Simulations led to a successful connection of two different aspects of the feasible static-wrench workspace for different actuator torque limits and different payload weights. Future work on this subject includes the design of a prototype to experimentally validate the approach. 
Table 1: Maximum values of input torques and optimal values for $\dot{\mathbf{q}}_{A}$ from the solution of the BVP.

\begin{tabular}{|l|c|c|c|}
\hline & $\begin{array}{c}\text { Max. Torques } \\
\text { Shooting method for BVP }\end{array}$ & $\begin{array}{c}\text { Max. Torques } \\
\text { Exciting trajectories }\end{array}$ & $\begin{array}{c}\text { Optimal Velocity } \\
\text { BVP }\end{array}$ \\
\hline & $|\boldsymbol{\tau}|_{\max }(\mathrm{Nm})$ & $|\boldsymbol{\tau}|_{\max }(\mathrm{Nm})$ & $\dot{\mathbf{q}}_{A}(\mathrm{rad} / \mathrm{s})$ \\
\hline Case 1 & {$[0.007,0.001]$} & {$[0.597,0.604]$} & {$[-7.257,4.494]$} \\
\hline Case 2 & {$[0.007,0.007]$} & {$[1.153,2]$} & {$[-10.052,10.179]$} \\
\hline Case 3 & {$[0.011,0.013]$} & {$[2.062,2.105]$} & {$[-10.916,10.625]$} \\
\hline Case 4 & {$[0.032,0.022]$} & {$[2.993,2.999]$} & {$[-11.802,12.649]$} \\
\hline
\end{tabular}

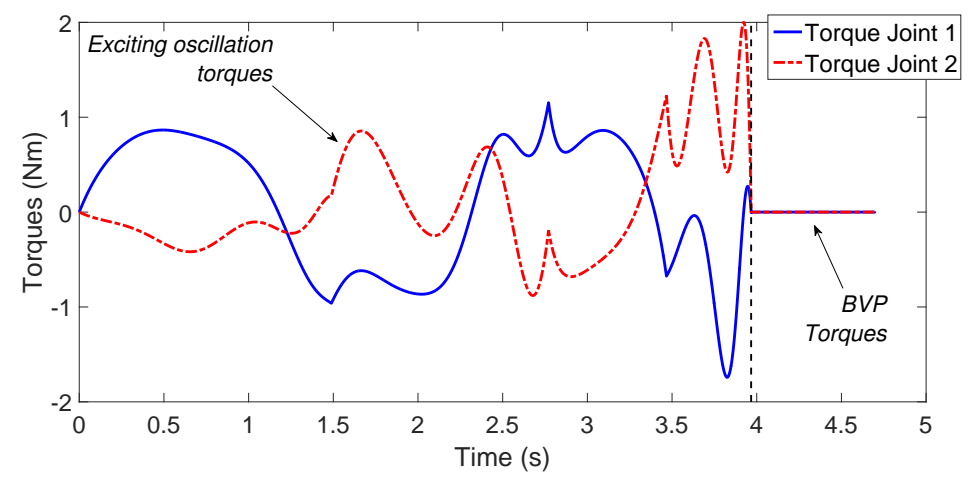

Fig. 3: Torques computed from the exciting-plus-BVP trajectory for the case 2.

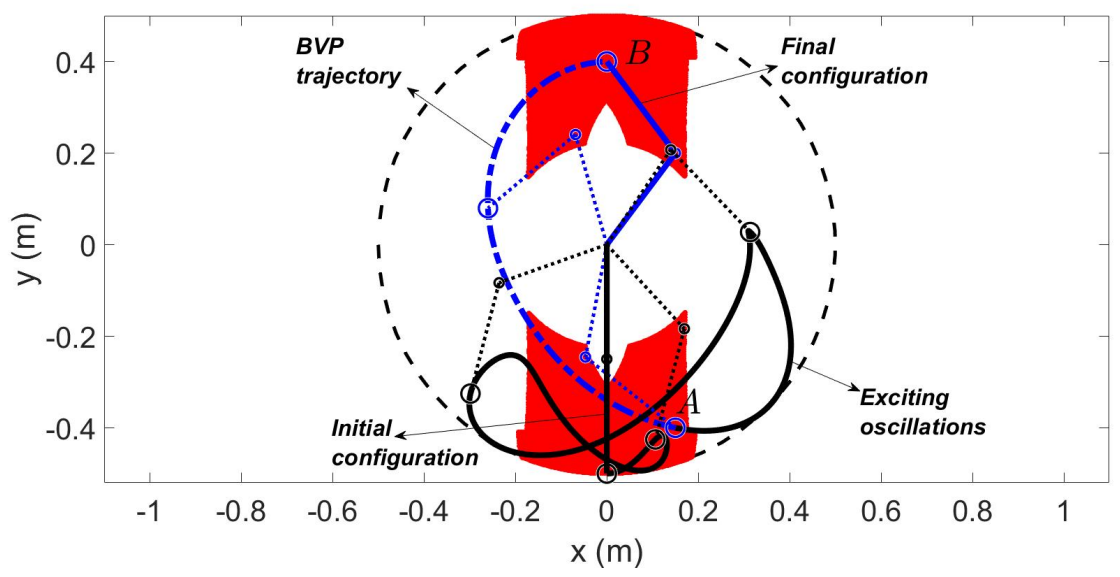

Fig. 4: Optimal trajectory for connecting two points by solving the BVP from boundaries of case 2 . $A$ and $B$ represent the boundary conditions of case 2 and they are joined thanks to the solution of the BVP. 


\section{Bibliography}

[1] M. Gouttefarde, J.-P. Merlet, and D. Daney: Wrench-feasible workspace of parallel cable-driven mechanisms. In Proc. IEEE International Conference on Robotics and Automation, Rome, Italy, 2007, pp. 1492-1497

[2] QJ. Duan and X. Duan: Workspace classification and quantification calculations of cable-driven parallel robots. In Adv. Mech. Eng. Volume 2014, Article ID 358727, 9 pages.

[3] M. Hiller, S. Fang, S. Mielczarek, R. Verhoeven, and D. Franitza: Design, analysis and realization of tendon-based parallel manipulators. In Mech. Mach. Theory, vol. 40, no. 4, pp. 429-445, April 2005.

[4] X. Jiang, E. Barnett, C. Gosselin: Periodic Trajectory Planning Beyond the Static Workspace for 6-DOF Cable-Suspended Parallel Robots. IEEE Transactions on Robotics, 2018, vol. 34, no. 4, pp. 1128-1140.

[5] C. Gosselin, P. Ren, and S. Foucault: Dynamic trajectory planning of a two-dof cable-suspended parallel robot. In Proc. IEEE International Conference on Robotics and Automation, St. Paul, MN, USA, May 1418, 2012, pp. 1476-1481.

[6] G. Mottola, C. Gosselin and M. Carricato: Dynamically feasible motions of a class of purely-translational cable-suspended parallel robots. In Mech. Mach. Theory, vol. 132, pp. 193-206, 2019.

[7] X. Jiang, C.Gosselin: Dynamic point-to-point trajectory planning of a three-DOF cable-suspended parallel robot. IEEE Transactions on Robotics 32(6), 1550-1557 (2016).

[8] J.P. Barreto, F.J.-F. Schöler and B. Corves: The concept of natural motion for pick and place operations. In: New advances in mechanisms, mechanical transmissions and robotics. Springer, pp. 89-98.

[9] W. Schiehlen, and N. Guse: Control of Limit Cycle Oscillations, In Proc. of the IUTAM Symposium on Chaotic Dynamics and Control of Systems and Processes in Mechanics, pp. 429-439, 2005.

[10] R. Balderas Hill, S. Briot, A. Chriette, and P. Martinet, 2018. Minimizing Input Torques of a High-Speed Five-Bar Mechanism by using Variable Stiffness Springs. Proceedings of the 22nd CISM-IFToMM Symposium on Robot Design, Dynamics, and Control (ROMANSY 2018), pp. 61-68, June 25-28, 2018, Rennes, France.

[11] E. Dombre and W. Khalil. Modeling, identification and control of robots. Hermes Sciences, Europe. Paris, 2002. ISBN 978-1-903996-66-9.

[12] S. M. Roberts and J. S. Shipman. Two-Point Boundary Value Problems: Shooting Methods. New York: Elsevier, 1972. ISBN 978-0444001023.

[13] R. Balderas Hill, S. Briot, A. Chriette, and P. Martinet, 2018. Increasing energy efficiency of high-speed parallel robots by using variable stiffness springs and optimal motion generation. Proceedings of the ASME 2018 IDETC/CIE 2018, pp. V05BT07A011 10 pages, August 26-29, 2018, Quebec City, Canada.

[14] A. L. C. Ruiz, S. Caro, P. Cardou, and F. Guay. ARACHNIS: Analysis of robots actuated by cables with handy and neat interface software. In Cable-Driven Parallel Robots, ser. Mechanisms and Machine Science, A. Pott and T. Bruckmann, Eds. Springer International Publishing, vol. 32, pp. 293-305. 